\title{
Prevalence of shedding and antibody to Coxiella burnetii in post-partum dairy cows and its association with reproductive tract diseases and performance: A pilot study
}

\author{
Marie-Ève Turcotte $^{\mathrm{a}}$, José Denis-Robichaud ${ }^{\mathrm{b}, 1}$, Jocelyn Dubuc ${ }^{\mathrm{b}}$, Josée Harel ${ }^{\mathrm{c}}$, \\ Donald Tremblay ${ }^{\mathrm{d}}$, Carl A. Gagnon ${ }^{\mathrm{c}, \mathrm{d}}$, Julie Arsenault ${ }^{\mathrm{a}, \mathrm{c}, *}$ \\ ${ }^{a}$ Groupe de recherche en épidémiologie des zoonoses et santé publique (GREZOSP), Faculté de médecine vétérinaire, Université de Montréal, 3200 Sicotte, St-Hyacinthe, \\ Québec, J2S 2M2, Canada \\ ${ }^{\mathrm{b}}$ Faculté de médecine vétérinaire, Université de Montréal, 3200 Sicotte, St-Hyacinthe, Québec, J2S 2M2, Canada \\ ${ }^{\mathrm{c}}$ Centre de Recherche en Infectiologie Porcine et Avicole (CRIPA-FQRNT), Faculté de médecine vétérinaire, Université de Montréal, 3200 Sicotte, St-Hyacinthe, Québec, \\ J2S 2M2, Canada \\ ${ }^{\mathrm{d}}$ Service de diagnostic, Faculté de médecine vétérinaire, Université de Montréal, 3200 Sicotte, St-Hyacinthe, Québec, J2S 2M2, Canada
}

\section{A R T I C L E I N F O}

\section{Keywords:}

Coxiella burnetii

Dairy cattle

Endometritis

Reproductive performance

Prevalence

\begin{abstract}
A B S T R A C T
The bacterium Coxiella burnetii has been associated with reproduction disorders in dairy cattle. A cross-sectional study was conducted in Québec, Canada, to estimate the prevalence of $C$. burnetii in dairy cows from $C$. burnetii RT-PCR-positive and/or ELISA-positive herds. As a secondary objective, the associations between C. burnetiipositivity and three reproductive outcomes (purulent vaginal discharge, cytological endometritis, and success at first service) were assessed. A total of 202 post-parturient dairy cows from nine herds were sampled at $35 \pm 7$ days in milk. Vaginal mucus and composite milk were collected from each cow and screened for the presence of C. burnetii by real-time PCR (RT-PCR) and ELISA, respectively. Purulent vaginal discharge and cytological endometritis were evaluated using a Metricheck device and a modified cytobrush, respectively. The first insemination postpartum was done following an ovulation synchronization protocol around 70 days in milk, and success at first service was recorded. Multilevel logistic regressions adjusted for parity were used to model purulent vaginal discharge, cytological endometritis and success at first service according to $C$. burnetii cow status. All 202 RT-PCR-assayed vaginal samples were $C$. burnetii-negative. A positive result for anti-C. burnetii antibodies detection in composite milk was obtained in 25/202 samples and a doubtful result in 4/202 samples. After adjustment for sampling weights, the 202 ELISA-assayed composite milk samples gave an estimated overall prevalence of $C$. burnetii positive cows of $12.9 \%(\mathrm{CI}=6.1-19.6 \%)$ and of doubtful cows of $1.4 \%(\mathrm{CI}=0.0-3.3$ $\%)$. The proportion of ELISA-positive cows was lower in first parity (0\%) compared to second (17.1 \%) or third parity cows (20.0\%). The associations between ELISA positivity and reproductive outcomes were not statistically significant, perhaps due to the limited sample size, but could be used as pilot estimate for large-scale studies investigating the impact of $C$. burnetii infection on reproduction disorders in dairy cattle.
\end{abstract}

\section{Introduction}

The bacterium Coxiella burnetii is the causative agent of $\mathrm{Q}$ fever (query fever), an important zoonotic disease in humans worldwide. Domestic ruminants are the main reservoir of the bacterium and can exhibit high prevalence of infection (Agerholm, 2013). In Québec, Canada, a study showed that $47.3 \%$ (35/74) of dairy herds were positive to C. burnetii based on serology and RT-PCR from bulk tank milk samples (Turcotte, 2015).

There is substantial evidence of an association between $C$. burnetii

\footnotetext{
* Corresponding author at: Groupe de recherche en épidémiologie des zoonoses et santé publique (GREZOSP), Faculté de médecine vétérinaire, Université de Montréal, 3200 Sicotte, St-Hyacinthe, Québec, J2S 2M2, Canada.

E-mail addresses: me.turcotte@yahoo.ca (M.-È. Turcotte), josedr@hotmail.ca (J. Denis-Robichaud), jocelyn.dubuc@umontreal.ca (J. Dubuc), josee.harel@

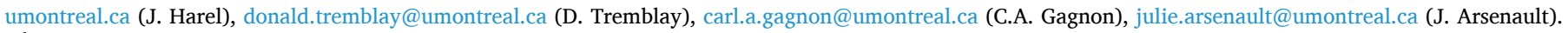

1 Independent researcher, Amqui, Québec, G5J 2N5, Canada.
} 
infection in dairy cattle and sporadic abortion (Agerholm, 2013). However, $C$. burnetii infection has been inconsistently associated with metritis, endometritis, infertility and retained placenta, with some studies lacking adequate controls for evaluating the impact of the infection (López-Gatius et al., 2012; Agerholm, 2013; García-Ispierto et al., 2013; Freick et al., 2017; De Biase et al., 2018). Following the repeated findings of a negative impact of purulent vaginal discharge and cytological endometritis on dairy cattle reproduction (McDougall et al., 2011; Denis-Robichaud and Dubuc, 2015a), it becomes relevant to revisit earlier findings based on the hypothesis that optimal diagnostic criteria for these two conditions would increase the ability to detect potential associations with $C$. burnetii positivity. A better understanding of the impacts of $C$. burnetii positivity on reproductive performances in dairy cattle is essential for evaluating the cost-benefits of control measures such as vaccination programs in a farm profitability and public health perspectives.

Our study was conducted to estimate the animal-level prevalence of C. burnetii in dairy cows from positive herds. As a secondary objective, the associations between $C$. burnetii positivity and purulent vaginal discharge, cytological endometritis, and reproductive performance were assessed as a pilot opportunity.

\section{Materials and methods}

\subsection{Data collection}

A cross-sectional study was conducted using a convenient sample of dairy cattle herds originally recruited for a randomized clinical trial on the efficacy of intrauterine infusion of cephapirin (Denis-Robichaud and Dubuc, 2015b). Three herds with a C. burnetii-ELISA- or -RT-PCR-positive bulk tank milk sample established in a concurrent study (Turcotte, 2015) were first selected. To reach the target sample size of 200 cows, cows from 9 additional herds with unknown $C$. burnetii status were -ELISA- and -RT-PCR-assayed in milk and vaginal samples, respectively (see Section 2.2). Only cows from herds with at least one C. burnetii-ELISA- or -RT-PCR-positive sampled cows were included in the study. The sample size of 200 cows was calculated to estimate the prevalence of positive cows with a precision of $5 \%$ and a confidence level of $95 \%$, given an expected prevalence of $16 \%$ (Muskens et al., 2011); we did not consider herd clustering in sample size calculation in the absence of prior data and expected limited variability in prevalence within $C$. burnetii positive farms. From selected herds, all cows that calved during the study period were sampled at $35 \pm 7$ days in milk. A vaginal mucus sample was first collected using a sterile BD Falcon SWUBE (Becton Dickinson, Oakville, ON, Canada). The presence of purulent vaginal discharge was evaluated with the Metricheck device (Simcro, Lawrence, KS, USA) using a cut-off $\geq 4$ indicative of a purulent discharge or worse (Denis-Robichaud and Dubuc, 2015a). Cytological endometritis was defined as the presence of $\geq 6 \%$ of polymorphonuclear cells in endometrial smear collected with the modified cytobrush technique (Denis-Robichaud and Dubuc, 2015a). The evaluation of purulent vaginal discharge and cytological endometritis were performed blindly to ELISA and RT-PCR testing. A composite milk sample was aseptically collected from each cow. First insemination of all enrolled cows was synchronized around 70 days in milk using Double-Ovsynch or Presynch-Ovsynch (systematic use of the same protocol within herds). Parity number, success at first service, and herd size data were collected.

\subsection{Laboratory analyses}

The vaginal mucus and milk samples were kept on ice and sent to the laboratory within $12 \mathrm{~h}$ of collection. The vaginal mucus samples were tested by RT-PCR as described elsewhere (Klee et al., 2006). The milk samples were assayed with the ID Screen ${ }^{\circledR} \mathrm{Q}$ Fever Indirect Multi-species ELISA kit (ID.Vet, Grabels, France) according to the manufacturer's instructions. Optical density ratio between sample and positive control (S/P) was used for interpretation: $<40 \%$ as negative, $40 \%-50 \%$ as doubtful, $>50 \%$ as positive.

\subsection{Statistical analyses}

All statistical procedures were performed in SAS software version 9.3 (SAS Institute Inc., Cary, NC, USA) using the cow as the unit of interest. Only cows from herds with at least one cow positive to ELISA (S/P > 50 $\%)$ or RT-PCR were kept for the study. Apparent prevalence of C. burnetii-positive cows for RT-PCR with $95 \%$ exact confidence intervals (CI) were estimated (due to the absence of positive sample, no adjustment for sampling design was used). Apparent prevalence of C. burnetii -positive or doubtful cows with $95 \%$ confidence intervals (CI) were estimated for ELISA results, adjusted for farm clustering and sampling weights. Only apparent prevalences were reported due to the absence of validated sensitivity and specificity estimates for the diagnostic tests used (Guatteo et al., 2011). Considering the low proportion of ELISA-doubtful results, and absence of a priori information to help classify them, they were excluded from further statistical analyses. The impact of parity group on $C$. burnetii ELISA positivity was analyzed using exact chi-square, followed by post-hoc pairwise testing with Bonferroni adjustment. This approach was chosen given the presence of a zero-count cell in the contingency table for this analysis, and absence of a statistically significant association between herd and ELISA positivity in preliminary analyses (chi-square test, $P=0.22$ ). Multilevel logistic regression models with random intercept for herds were used to evaluate the association between $C$. burnetii positivity and the following outcomes: purulent vaginal discharge, cytological endometritis, and success at first service as the outcome, with parity groups forced into the models as a potential confounder. Odds ratios (OR) were used to present results. Alpha value was set at $5 \%$.

\section{Results}

Between August 27th and October 31st, 2012, 12 dairy herds were sampled. Three herds were excluded because no $C$. burnetii-positive cows were detected. Of the nine herds included in our study, three used a free stall housing system and six used a tie-stall barn. The herd size ranged from 65 to 220 cows (median $=90$ ). Between nine and 70 cows (median $=20$ ) were sampled per herd, for a total of 202 cows. Among the 196 cows with information available on parity, 62 (32\%) cows were in their 1st parity, 43 (22\%) in their 2nd and 91 (46\%) in their $\geq 3$ rd parity.

All 202 vaginal samples were $C$. burnetii-RT-PCR-negative, leading to an apparent prevalence of positive cows estimate of $0 \%(\mathrm{CI}=0.0-1.8 \%)$ for RT-PCR. Anti-C. burnetii antibodies were detected in 25 milk samples and four additional samples were doubtful (Fig. 1). Thus, the estimated prevalence of ELISA-positive cows was $12.9 \%(\mathrm{CI}=6.1-19.6 \%)$, and was of $1.4 \%$ (CI $=0.0-3.3 \%)$ for ELISA-doubtful cows.

An overall association between parity group and ELISA positivity was detected ( $P=0.001$, exact chi-square), with a proportion of C. burnetii-ELISA positive cows of $0 \%$ in 1 st parity $(\mathrm{n}=61), 17.1 \%$ in 2nd parity $(n=41)$ and $20.0 \%$ in 3rd parity $(n=90)$ cows. According to post-hoc tests, 1 st parity cows had a statistically significantly lower risk of positivity than 2nd or 3rd parity cows. No association with cow C. burnetii ELISA-positivity was observed for purulent vaginal discharge $(\mathrm{OR})=0.68, P=0.57)$, cytological endometritis $(\mathrm{OR}=1.0, P=0.95)$, or success at first service $(\mathrm{OR}=1.3, P=0.64)$ in multilevel logistic regression models adjusted for parity groups (Table 1 ).

\section{Discussion}

The estimated apparent prevalence of $C$. burnetii ELISA-positive cows in our study was lower than the $19.4 \%$ animal-level median apparent prevalence reported in a review conducted in cattle (Guatteo et al., 2011). In our study, only C. burnetii-positive herds were kept for prevalence estimation, whereas Guatteo et al. (2011) included all herds, 


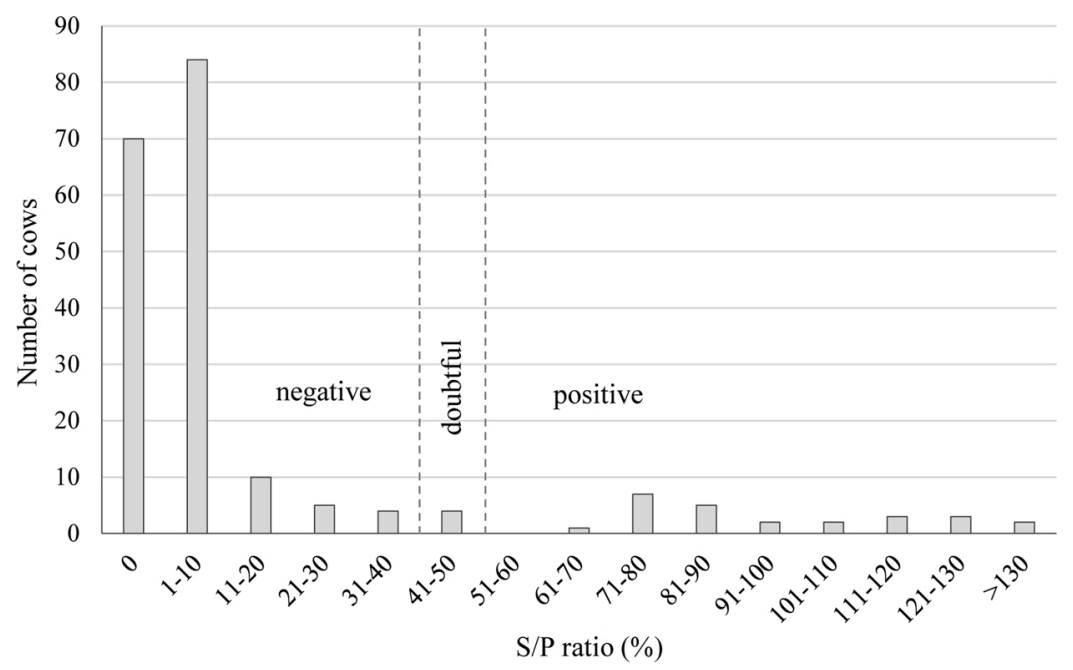

Fig. 1. Distribution of S/P ratio (\%) in 202 cows from Coxiella burnetii-positive (ELISA and/or RT-PCR) herds, Québec, Canada, 2012.

Table 1

Multivariable analyses of the effect of Coxiella burnetii ELISA positivity and parity on three reproductive outcomes in dairy cows, Québec, Canada, 2012.

\begin{tabular}{|c|c|c|c|c|c|}
\hline \multicolumn{6}{|c|}{ a. Purulent vaginal discharge ( $\mathrm{n}=191 \operatorname{cows}^{\mathrm{a}}$ from 9 herds) } \\
\hline \multirow[b]{2}{*}{ Variables } & \multirow[b]{2}{*}{$\begin{array}{l}\text { Number } \\
\text { of cows }\end{array}$} & \multirow{2}{*}{$\begin{array}{l}\text { Number of cows } \\
\text { (\%) with purulent } \\
\text { vaginal discharge }\end{array}$} & \multicolumn{3}{|l|}{ Odds ratio } \\
\hline & & & Estimate & $95 \%$ CI & $\begin{array}{l}P \text { - } \\
\text { value }\end{array}$ \\
\hline \multicolumn{6}{|c|}{ Coxiella burnetii ELISA status } \\
\hline Positive & 24 & $3(12.5)$ & 0.68 & $0.18-2.6$ & 0.57 \\
\hline \multirow{2}{*}{\multicolumn{6}{|c|}{ Parity }} \\
\hline & & & & & \\
\hline $1^{\mathrm{st}}$ & 61 & $11(18.0)$ & Ref. & & \\
\hline $2^{\text {nd }}$ & 41 & $4(9.8)$ & 0.52 & $0.15-1.8$ & 0.30 \\
\hline $3^{\text {rd }}$ & 89 & $17(19.1)$ & 1.15 & $0.48-2.7$ & 0.76 \\
\hline \multicolumn{6}{|c|}{ b. Cytological endometritis ( $\mathrm{n}=190$ cows $^{\mathrm{b}}$ from 9 herds) } \\
\hline \multirow[b]{2}{*}{ Variables } & \multirow[b]{2}{*}{$\begin{array}{l}\text { Number } \\
\text { of cows }\end{array}$} & \multirow{2}{*}{$\begin{array}{l}\text { Number of cows } \\
\text { (\%) with } \\
\text { cytological } \\
\text { endometritis }\end{array}$} & \multicolumn{3}{|l|}{ Odds ratio } \\
\hline & & & Estimate & $95 \% \mathrm{CI}$ & $\begin{array}{l}P \text { - } \\
\text { value }\end{array}$ \\
\hline \multicolumn{6}{|c|}{ Coxiella burnetii ELISA status } \\
\hline Positive & 25 & $10(40.0)$ & 1.0 & $0.41-2.6$ & 0.95 \\
\hline Negative & 165 & $53(32.1)$ & Ref. & & \\
\hline \multicolumn{6}{|l|}{ Parity } \\
\hline $1^{\text {st }}$ & 61 & $12(19.7)$ & Ref. & & \\
\hline $2^{\text {nd }}$ & 39 & $12(30.8)$ & 1.8 & $0.70-4.7$ & 0.22 \\
\hline $3^{\text {rd }}$ & 90 & $39(43.3)$ & 3.1 & $1.4-6.8$ & $<0.01$ \\
\hline \multicolumn{6}{|c|}{ c. Success at first service ( $\mathrm{n}=177$ cows $^{\mathrm{c}}$ from 9 herds) } \\
\hline \multirow[b]{2}{*}{ Variables } & \multirow{2}{*}{$\begin{array}{l}\text { Number of } \\
\text { cows }\end{array}$} & \multirow{2}{*}{$\begin{array}{l}\text { Number of cows } \\
(\%) \text { with success at } \\
\text { first service }\end{array}$} & \multicolumn{3}{|l|}{ Odds ratio } \\
\hline & & & Estimate & $95 \%$ CI & $\begin{array}{l}P \text { - } \\
\text { value }\end{array}$ \\
\hline \multicolumn{6}{|c|}{ Coxiella burnetii ELISA status } \\
\hline Positive & 24 & $9(37.5)$ & 1.3 & $0.48-3.3$ & 0.64 \\
\hline Negative & 153 & $50(32.7)$ & Ref. & & \\
\hline \multicolumn{6}{|l|}{ Parity } \\
\hline $1^{\mathrm{st}}$ & 60 & $19(31.7)$ & Ref. & & \\
\hline $2^{\text {nd }}$ & 38 & $18(47.4)$ & 1.9 & $0.79-4.4$ & 0.16 \\
\hline $3^{\text {rd }}$ & 79 & $22(27.9)$ & 0.79 & $0.37-1.7$ & 0.55 \\
\hline
\end{tabular}

${ }^{a}$ From the 202 cows in the study, 4 with doubtful ELISA result, 6 with missing value for parity and 1 with missing value for purulent vaginal discharge were excluded.

${ }^{\mathrm{b}}$ From the 202 cows in the study, 4 with doubtful ELISA result, 6 with missing value for parity and 2 with missing value for cytological endometritis were excluded.

${ }^{c}$ From the 202 cows in the study, 4 with doubtful ELISA result, 6 with missing value for parity and 15 with missing value for success at first service were excluded. which even further support the hypothesis of a low level of infection in Quebec's dairy cattle herd. On the other hand, as a decrease in antibody levels in milk samples has been reported in the weeks following calving, our prevalence might be underestimated (García-Ispierto et al., 2011; Walraph et al., 2018). The prevalence of ELISA-positive cows was very low among 1st parity cows, suggesting a lower risk of exposure before first parturition, perhaps because heifers could be kept apart from adult cows in this production system. Older cows were more likely to be ELISA-positive, as previously reported by Böttcher et al. (2011).

No significant effect of cow ELISA positivity was found on purulent vaginal discharge, endometritis, and success at first service. Despite the fact that $C$. burnetii was reported to cause inflammation in the bovine uterus and placenta, there is no strong scientific evidence supporting a negative impact of $C$. burnetii infection on metritis or endometritis in dairy cattle populations (Agerholm, 2013; De Biase et al., 2018). A recent study reported an absence of association between many parameters of reproduction and seropositivy in primiparous cows (Freick et al., 2017). A study has even observed that seropositive cows were at a lower risk of endometritis (García-Ispierto et al., 2013). Likewise, while Literak and Kroupa (1998) did not find any association between dairy cattle herd seropositivity and conception rate to first service, García-Ispierto et al. (2013) observed that $C$. burnetii shedding was associated with delayed conception among seronegative cows. On the other hand, Khalili et al. (2012) showed an association between anti-C. burnetii antibodies in cattle and reproductive disorders. Besides these disorders, C. burnetii detection by molecular method was reported in only $1 \%$ of abortion cases in dairy cattle in Quebec (Gagnon, 2013). Overall, these results suggest only minimal effects of C. burnetii infection on dairy cattle farm productivity. Therefore, it highlights the policy challenge of cost sharing between the industry and public health for implementing control measures on farm that would mostly benefit public health. In our study, C. burnetii genes had been previously detected using molecular techniques in bulk milk in two of the sampled herds (Turcotte, 2015), and the presence of ELISA-positive cows here suggests that the infection had been circulating in all herds included in our analyses. Nevertheless, the impact of the bacterial infection could depend on the state of the infection, acute or chronic, and could vary according to the animal immunity. Overall, our ELISA-positive cows had relatively low serological reactions when compared to other dairy cattle herds in which C. burnetii was previously identified by PCR on vaginal mucus from aborted cows (Guatteo et al., 2007). The absence of detected shedding may also imply that the bacterium was not actively circulating in the herd at time of the study. It is also possible that different $C$. burnetii strains could be involved, as previously hypothesized (Samuel et al., 1985). 
The lack of association between $C$. burnetii infection and reproductive performance in our study could also be due to some design limitations. First, there may have been early culling of $C$. burnetii-infected cows with impaired reproduction performances, as previously hypothesized (García-Ispierto et al., 2011). Also, infection with C. burnetii could have led to endometritis, followed by clearance of the bacteria prior to sampling while the inflammation could still be detected, as previously suggested for other bacteria (McDougall et al., 2011). The cross-sectional nature of our study does not indicate the timing of this infection which would likely influence the impact on reproductive diseases. Finally, a lack of statistical power is very likely considering that the sample size was determined for another purpose. Nevertheless, this pilot study provides useful preliminary estimates for planning further larger size longitudinal studies.

\section{Conclusions}

At 35 days in milk, none of the 202 vaginal mucus sample was positive to $C$. burnetii, even if 25 were collected from $C$. burnetii ELISApositive cow. Primiparous were less likely to be $C$. burnetii ELISApositive than multiparous cows in herds known to be positive to the bacteria, suggesting the infection might occur after the first gestation. Coxiella burnetii ELISA positivity was not associated with reproductive disease and performance in the studied herds, but larger scale longitudinal studies are needed to confirm the absence of association. Further studies should be conducted to elucidate the herd, individual and C. burnetii strain-related factors associated with previously reported negative impact of this infection.

\section{Acknowledgements}

We greatly acknowledge the participating producers for their time and contribution to this study, Jean-Philippe Pelletier for his contribution to the field work, as well as Brigitte Bousquet for laboratory analyses conducted at the Veterinary Virology Diagnostic Laboratory (FMV, UdeM). We are thankful to Jean-Charles Côté for critical reading of the manuscript. This study was funded by the Green Fund of the Ministère de la Santé et des Services sociaux du Québec within the framework of Action 21 of the 2006-2012 Climate Change Action Plan (CCAP) with the financial support of the Ministère de l'Agriculture, des Pêcheries et de l'Alimentation du Québec.

\section{References}

Agerholm, J.S., 2013. Coxiella burnetii associated reproductive disorders in domestic animals - a critical review. Acta Vet. Scand. 55, 13. https://doi.org/10.1186/1751 0147-55-13.

Böttcher, J., Vossen, A., Janowetz, B., Alex, M., Gangl, A., Randt, A., Meier, N., 2011. Insights into the dynamics of endemic Coxiella burnetii infection in cattle by application of phase-specific ELISAs in an infected dairy herd. Vet. Microbiol. 151, 291-300. https://doi.org/10.1016/j.vetmic.2011.03.007.

De Biase, D., Costagliola, A., Del Piero, F., Di Palo, R., Coronati, D., Galiero, G., Uberti, B. D., Lucibelli, M.G., Fabbiano, A., Davoust, B., Raoult, D., Paciello, O., 2018. Coxiella burnetii in infertile dairy cattle with chronic endometritis. Vet. Pathol. 55, 539-542. https://doi.org/10.1177/0300985818760376.

Denis-Robichaud, J., Dubuc, J., 2015a. Determination of optimal diagnostic criteria for purulent vaginal discharge and cytological endometritis in dairy cows. J. Dairy Sci. 98, 6848-6855. https://doi.org/10.3168/jds.2014-9120.

Denis-Robichaud, J., Dubuc, J., 2015b. Randomized clinical trial of intrauterine cephapirin infusion in dairy cows for the treatment of purulent vaginal discharge and cytological endometritis. J. Dairy Sci. 98, 6856-6864. https://doi.org/10.3168/ jds.2014-9129.

Freick, M., Enbergs, H., Walraph, J., Diller, R., Weber, J., Konrath, A., 2017. Coxiella burnetii: serological reactions and bacterial shedding in primiparous dairy cows in an endemically infected herd-impact on milk yield and fertility. Reprod. Domest. Anim. 52, 160-169. https://doi.org/10.1111/rda.12878.

Gagnon, C.A., 2013. Le diagnostic des maladies infectieuses virales bovines : du présent au futur [Diagnosis of Viral Infectious Diseases in Cattle: From Past to Present]. Congrès Vétérinaire Québécois, Saint-Hyacinthe, Quebec, Canada.

García-Ispierto, I., Almeria, S., López-Gatius, F., 2011. Coxiella burnetii seropositivity is highly stable throughout gestation in lactating high-producing dairy cows. Reprod. Domest. Anim. 46, 1067-1072. https://doi.org/10.1111/j.1439-0531.2011.01788. $\mathrm{x}$.

García-Ispierto, I., López-Helguera, I., Tutusaus, J., Serrano, B., Monleón, E., Badiola, J. J., López-Gatius, F., 2013. Coxiella burnetii shedding during the peripartum period and subsequent fertility in dairy cattle. Reprod. Domest. Anim. 48, 441-446. https:// doi.org/10.1111/rda.12095.

Guatteo, R., Beaudeau, F., Joly, A., Seegers, H., 2007. Coxiella burnetii shedding by dairy cows. Vet. Res. 38, 849-860. https://doi.org/10.1051/vetres:2007038.

Guatteo, R., Seegers, H., Taurel, A.F., Joly, A., Beaudeau, F., 2011. Prevalence of Coxiella burnetii infection in domestic ruminants: a critical review. Vet. Microbiol. 149, 1-16. https://doi.org/10.1016/j.vetmic.2010.10.007.

Khalili, M., Sakhaee, E., Babaei, H., 2012. Frequency of anti-Coxiella burnetii antibodies in cattle with reproductive disorders. Comp. Clin. Path. 21, 917-919. https://doi. org/10.1007/s00580-011-1199-2.

Klee, S.R., Tyczka, J., Ellerbrok, H., Franz, T., Linke, S., Baljer, G., Appel, B., 2006. Highly sensitive real-time PCR for specific detection and quantification of Coxiella burnetii. BMC Microbiol. 6, 2. https://doi.org/10.1186/1471-2180-6-2.

Literak, I., Kroupa, L., 1998. Herd-level Coxiella burnetii seroprevalence was not associated with herd-level breeding performance in Czech dairy herds. Prev. Vet. Med. 33, 261-265. https://doi.org/10.1016/S0167-5877(97)00034-2.

López-Gatius, F., Almeria, S., García-Ispierto, I., 2012. Serological screening for Coxiella burnetii infection and related reproductive performance in high producing dairy cows. Res. Vet. Sci. 93, 67-73. https://doi.org/10.1016/j.rvsc.2011.07.017.

McDougall, S., Hussein, H., Aberdein, D., Buckle, K., Roche, J., Burke, C., Mitchell, M., Meier, S., 2011. Relationships between cytology, bacteriology and vaginal discharge scores and reproductive performance in dairy cattle. Theriogenology 76, 229-240. https://doi.org/10.1016/j.theriogenology.2010.12.024.

Muskens, J., van Engelen, E., van Maanen, C., Bartels, C., Lam, T.J.G.M., 2011. Prevalence of Coxiella burnetii infection in Dutch dairy herds based on testing bulk tank milk and individual samples by PCR and ELISA. Vet. Rec. 168, 79. https://doi. org/10.1136/vr.c6106.

Samuel, J.E., Frazier, M.E., Mallavia, L.P., 1985. Correlation of plasmid type and disease caused by Coxiella burnetii. Infect. Immun. 49, 775-779.

Turcotte, M.-E., 2015. Prévalence et facteurs de risque de l'infection par Coxiella burnetii chez les ruminants d'élevage au Québec [Prevalence of and Risk Factors Associated with Coxiella burnetii in Domestic Ruminants in Quebec]. MSc Thesis. Faculty of Veterinary Medicine, Université de Montréal, Quebec, Canada, p. 220.

Walraph, J., Zoche-Golob, V., Weber, J., Freick, M., 2018. Decline of antibody response in indirect ELISA tests during the periparturient period caused diagnostic gaps in Coxiella burnetii and BVDV serology in pluriparous cows within a Holstein dairy herd. Res. Vet. Sci. 118, 91-96. https://doi.org/10.1016/j.rvsc.2018.01.018. 